\title{
A Simple Resonance Method to Investigate Dielectric Constant of Low Loss Substrates for Smart Clothing
}

\author{
Hemin Ismael Azeez, ${ }^{1}$ Wen-Shan Chen, ${ }^{2}$ Chih-Kuang Wu, ${ }^{1}$ \\ Chien-Min Cheng, ${ }^{2}$ and Hung-Chi Yang ${ }^{1 *}$ \\ ${ }^{1}$ Department of Electrical Engineering, Southern Taiwan University of Science and Technology, \\ No. 1, Nan-Tai Street, Yungkang Dist., Tainan City 710, Taiwan \\ ${ }^{2}$ Department of Electronic Engineering, Southern Taiwan University of Science and Technology, \\ No. 1, Nan-Tai Street, Yungkang Dist., Tainan City 710, Taiwan
}

(Received November 15, 2017; accepted January 15, 2018)

Keywords: wearable antenna, fabric characterization, dielectric constant, smart clothes

Wireless body area networks (WBANs), because of their widespread applications in health care and industrial development, have an important share of the recent advancements of the Internet of things (IoT). The term "wireless" is a key element of any new user-friendly wearable technological devices in health monitoring. This concept is reinforced by the utilization of wearable antennas and ultimately leads to the integration of technique and clothes; therefore the concept of smart clothing has been introduced. Wearable antennas are different because they are planar, durable, and user friendly. In this regard, the most prevalent type of antenna that has variety of forms and patterns is the microstrip antenna. Planar antennas in general need flexible and thin substrates that enable them to be mounted on textiles. Consequently, materials such as felt, polyester, and denim are excellent candidates for the development of wearable antennas. The materials are known for having less loss in comparison to conventional substrates. However, the electromagnetic properties of such regular fabrics are not well known and are none of fabric manufacturer's concern. Therefore, a very simple technique to characterize the electromagnetic properties of sample fabrics as antenna substrates for wireless body communications in this study is proposed. Because microstrip antennas are less dependent on the imaginary part of the complex dielectric constant of the substrates, the unique method presented in this paper helps researchers determine the real part of the dielectric permittivity straightforwardly and without advanced knowledge of the substrate. The method can be considered as an example of applications of antennas to characterizing electromagnetic properties of materials. The structure of the method involves square-patch antennas that are directly fed at the center of the patch. The lowest resonance mode of the patch antenna is the key parameter in the process. Step-by-step procedures and materials used in this study to develop the method are explained and discussed. The stability and accuracy of the method is evaluated using simulations and measurements.

*Corresponding author: e-mail: hugiyang@stust.edu.tw http://dx.doi.org/10.18494/SAM.2018.1828 


\section{Introduction}

Improving one's health by real-time monitoring of the human body's information and increasing life expectancy are basically the main purposes of wearing a wearable device. Thus, a variety of sensors, such as body temperature, heart rate, blood pressure, and respiration rate sensors, are used to collect important information about the user's activities or one's physiological condition. Sensors with wearable wireless health care devices can be mounted on or integrated into flexible fabrics, such as denim, felt, and clothes. This provides a costeffective and user-friendly environment for the users, and data acquisition, and analysis by health care clinics at a distance.

Studying substrates of antennas whose radiating elements are considered to be arranged and placed in one plane is of great interest. Substrates have a huge impact on the ability to integrate antennas into clothing to wireless body area networks (WBAN) or the Internet of things (IoT), so that smart clothing can communicate with other technologies. ${ }^{(1)}$ To be user friendly and effective, wearable antennas are required to have flexible structures. This requirement is accomplished by choosing the proper fabric as the substrate, such as denim, felt, or polyesters. ${ }^{(2)}$ The electromagnetic properties of the substrate and its thickness crucially affect the performance of planar antennas in terms of efficiency and impedance bandwidth. ${ }^{(3)}$ Therefore, the need to characterize and determine the electromagnetic properties of these substrates is vital. Varieties of substrates are employed for planar dipoles, monopoles, and microstrip antennas. Accordingly, substrates are mainly categorized by their dielectric constant and the loss factor. Substrates with low dielectrics and high dielectric constants have been reported in literature. ${ }^{(4)}$ In addition, the substrates used in smart clothing are mainly classified as lossless and lossy materials. ${ }^{(5)}$ Wearable planar antennas have very important applications in industry and telemedicine. Initially, the paper introduces a simple technique to measure and determine the dielectric constant in low loss fabrics used in developing wearable planar textile antennas. A resonance method based on square-patch antennas is exploited to characterize the real part of the dielectric permittivity. The method is validated using an FR4 substrate with known dielectric properties. Finally, various tasks are carried out to further validate and test the accuracy of the proposed method.

\subsection{Fabrics}

Fabrics chosen for wearable antenna development are classified into dielectric and conductive fabrics.

1. Electrically conductive fabrics (electro-textiles): This type of fabric is mainly used to model a radiating element and should meet the following requirements to be functionally valid.

a. The resistance variance of the sheet must be as small as possible (homogeneous) over the area of the antenna. ${ }^{(2)}$

b. To be user-friendly and easily applied to different forms, the fabric must be elastic and flexible.

2. Normal dielectric fabrics: Synthetic dielectrics have been used as substrates the wearable 
antennas made from are denim fabrics, polycot, polyesters, and woven and washed cottons. Textiles are very permeable to airflow. Therefore, owing to the presence of air between the fibers of the fabric, the dielectric permittivity always lies between 1 and $2 .^{(3)}$

\subsection{Overview of dielectric constant characterization techniques}

A variety of techniques and algorithms are available in the literature to characterize the electromagnetic properties of materials. The techniques can be generally split into two categories: resonant and nonresonant techniques. ${ }^{(4)}$ Resonant techniques are used when accurate knowledge of a material's electromagnetic properties at a specific frequency or at several discrete frequencies is needed. Meanwhile, non-resonant methods are exploited when general knowledge of a material's electromagnetic properties over a range of frequencies is desired. According to the resonator technique, the device under study becomes the resonator itself or a part of a resonant structure. Accuracy and sensitivity are the key advantages of resonator methods. The resonant properties of a resonant structure are used to extract the material properties.

With regard to nonresonant methods, the characteristic impedance and wave velocities of a propagated wave are used to determine the material properties. When a wave propagates from one medium to another, information about the reflected waves and transmitted waves at the interface between the two media is used to extract the electromagnetic properties of the material. ${ }^{(6)}$ Characterizing electromagnetic properties of the materials is always a tradeoff. Different methods to characterize electromagnetic properties of materials use different approaches. Some are more complicated than others. In general, several facts provide information about which method is the best.

- The frequency of interest (single frequency or a range of frequencies)

- Type of material (lossy, low loss, lossless) and expected permittivity value

- The required measurement accuracy (accurate, less accurate)

- Material properties (homogeneous, isotropic)

- The form of material (liquid, powder, solid, sheet)

- Restrictions on the material size

- Conducting, partially conducting, or fully dielectric

- The desired temperature during measurement

Because some materials are too expensive to acquire or their chemical characteristics are easily affected, all the points listed above need to be considered when selecting a measuring technique. As mentioned, many techniques are proposed in the literature to characterize the electromagnetic properties of different materials. Several techniques are employed to determine both the dielectric constant and the dissipation factor. A few techniques are proposed to find only the dielectric constant of materials used in applications in which the dissipation factor of the material has little effect on performance. ${ }^{(5)}$ To measure the dielectric constant and the dissipation factor of a printed circuit board accurately over a wide frequency range, a two-port microstrip ring resonator is used and the temperature of the printed circuit board (FR-4) is taken into considerations. ${ }^{(7)}$ A microwave method was proposed by Hasar and Westgate to determine 
the complex permittivity of low loss materials. ${ }^{(8)}$ The method used measured S-parameters $\left(S_{11}\right.$, $S_{12}, S_{13}$, and $S_{22}$ ) to extract the complex permittivity of two different samples, polyvinyl-chloride (PVC) and Plexiglas. A non-resonant microwave algorithm has been proposed for determining complex permittivity of low loss materials from the measured transmission S-parameters over a wide frequency range. ${ }^{(9)}$ Both complex permittivity and permeability of linear low loss materials were extracted from the measured scattering coefficients when a coaxial transmission line method was used. ${ }^{(10)}$

Open-ended coaxial probes were employed in a coaxial line reflection method to accurately characterize the properties of materials. ${ }^{(1)}$ The method can be categorized in terms of two models. In the first model, a coaxial probe terminated in layered dielectric materials of finite thickness. ${ }^{(12-15)}$ Here, the sample of the dielectric material is backed by either free space or a finite metal sheet as seen in Fig. 1. In the second model a coaxial line probe exited into a dielectric sample. This can be considered as an attempt to apply monopole antennas to determine the dielectric properties of materials. ${ }^{(16,17)}$ Very short coaxial probes may be broadly used as monopole radiators to characterize biological samples for medical purposes as shown in Fig. 2. Simplicity in the fabrication process has made this method widely used in material property characterization.

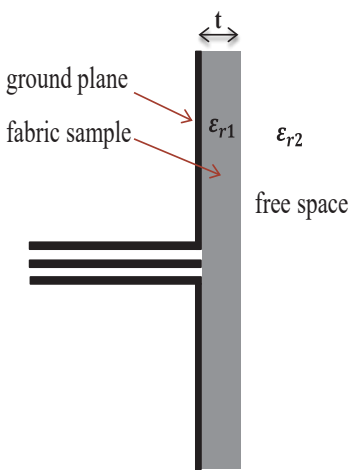

(a)

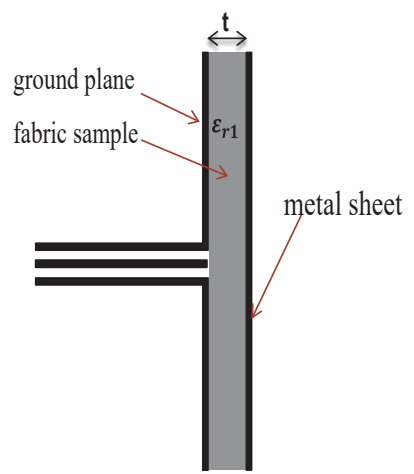

(b)

Fig. 1. (Color online) Structure of coaxial line reflection method backed by (a) free space or (b) metal sheet.

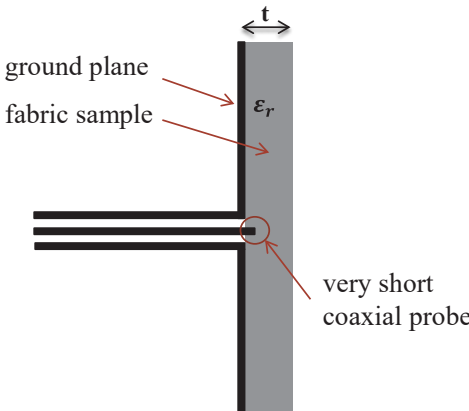

(a)

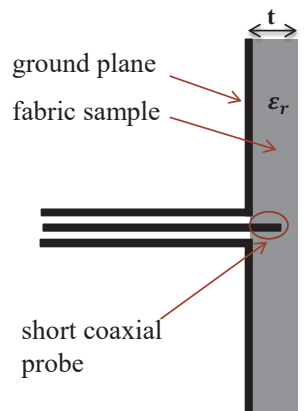

(b)

Fig. 2. (Color online) Geometry of monopole antennas used in material characterizations: (a) very short coaxial probe and (b) short coaxial probe. 
In research carried out by Chen et al. ${ }^{(18)}$ the concept of applying antennas to material property characterization has also been used. Using a rectangular patch radiator on fabric based substrates is an example of a resonant method to extract the dielectric constant of a fabric. The proposed method works based on assumptions regarding the dielectric constants of the fabric given in literature. Our research introduces a simple unique approach that can be used by researchers with minimal facilities to obtain accurate dielectric permittivity values of low loss fabric substrates targeted for use in smart clothing applications.

\section{Description of the Proposed Approach}

The proposed algorithm presents an example of applying antennas to the determination of material characteristics.

\subsection{Structure}

The geometry of the proposed technique is equivalent to a combined version of both the structures shown in Figs. 1 and 2. An open-ended coaxial probe method, in which the substrate being tested terminates the probe and is backed by a metal sheet, is shown in Fig. 1. The excited coaxial probe method in which the probe enters the substrate, and known as monopole antenna radiator is shown in Fig. 2. The method is an example of using an antenna to extract the real part of the permittivity of low loss fabrics used in ongoing studies in the field of wearable technologies and smart clothing. As is shown in Fig. 3, the structure of the proposed method is that of one type of planar antenna called a patch antenna. The microstrip patch is square and fed by the coaxial probe at its center. The structure is applied to determine the real part of the permittivity of homogenous fabric with a finite thickness and used in new smart clothing wearable technologies. The method is based on resonance frequency and uses approximate formulas for a rectangular patch antenna to extract the real part of dielectric permittivity of the medium. The imaginary part of the fabric's dielectric permittivity is not discussed and is only highlighted here because fabrics in general are low loss materials and the basic approaches to wearable antennas in this regard mostly deal with microstrip patch antennas which are planar and whose resonance frequencies only depend on the real part of the permittivity. ${ }^{(19-25)}$

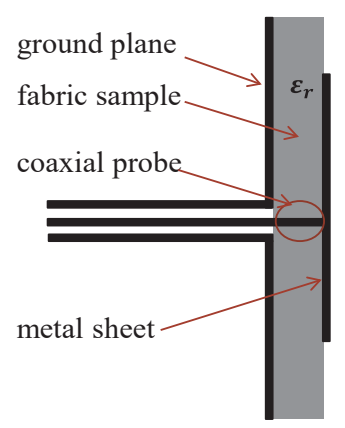

(a)

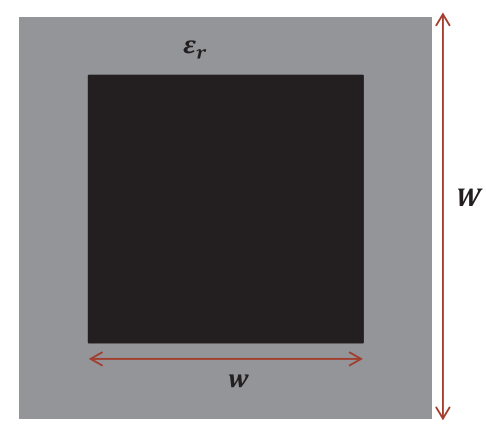

(b)

Fig. 3. (Color online) Geometry of the proposed method: (a) side view and (b) front view. 


\subsection{Simplified formulation}

In modelling and designing wearable antennas for wireless wearable technologies and smart clothing, low loss thin fabrics are normally preferred. Owing to its simplicity and accuracy in analysis, the rectangular patch antenna is the preferred configuration widely referred to in the literature. In this context, the transmission line model and cavity model are two widely used methods to analyze rectangular microstrip patch antennas. In this study, owing to the discrepancies between the effective electrical dimensions of a rectangular patch antenna in practice and its dimensions according to theoretical formulas, the principal transmission line model formulas used to analyze rectangular patch antennas have been simplified. ${ }^{(26)}$ The simplified versions of the formulas are considered the base equations for the proposed structure (square patch radiator fed at the center) shown in Fig. 3 which were used to extract accurate values from fabric of interest. In resonator methods, the dielectric constant is extracted from the dielectric resonator resonance frequency. However in this study, the dielectric constant was derived from the lowest resonance frequency (fundamental mode) of the square patch radiator as a function of the area of the radiating patch.

\subsection{Derived formulas}

1. Assume that the fabric sample being tested is uniform with finite thickness $(t)$.

2. The resonance frequency of the patch is $f_{r n}(\mathrm{~Hz}) ; n=1$, which is the lowest resonance frequency.

3. The patch is square, hence, the ground plane area $=W^{2}\left(\mathrm{~m}^{2}\right)$, the radiating patch area $=w^{2}\left(\mathrm{~m}^{2}\right)$, and $(W, w) \gg t$, assuming $w \leq W$.

4. For a rectangular patch, the effective patch length can be expressed as

$$
L_{e f f}=L+2 \Delta L=\frac{c_{0}}{2 f_{r n} \sqrt{\varepsilon_{\text {reff }}}},
$$

where $L$ is the physical patch length, $\Delta L$ is the extended length to reduce fringing effect, $\varepsilon_{\text {reff }}$ is effective real part of the dielectric constant, and $c_{0}(\mathrm{~m} / \mathrm{s})$ is the speed of light in a vacuum.

5. Assuming that the radiation patch width $w \cong 2 L_{\text {eff }}$, then from equation (1) the lowest resonant mode of the patch can be expressed as :

$$
f_{r 1}=\frac{c_{0}}{w \sqrt{\varepsilon_{r e f f}}}
$$

From Eq. (2),

$$
\varepsilon_{\text {reff }}=\left(\frac{c_{0}}{w f_{r 1}}\right)^{2} .
$$


From the transmission line model, and the analysis of fringing effect, let

$$
a=\sqrt{1+\frac{12 t}{w}}
$$

The dielectric constant is extracted from

$$
\varepsilon_{r}=\frac{2 a \varepsilon_{r e f f}-(a-1)}{(a+1)} .
$$

6. For the mean value of $\varepsilon_{r}$

$$
\varepsilon_{r(\text { mean })}=\frac{\sum_{i=1}^{N}\left(\varepsilon_{r i}\right)}{N}
$$

where $N$ is the number of measurements performed for different dimensions of the patch radiator or for different substrate thickness.

\subsection{Design and measurement procedures}

The dielectric constant of the fabric according to the method presented in our research can be more easily extracted and measured than from that proposed by Sankaralingam and Gupta. ${ }^{(5)}$ In our method, the effective permittivity of the material used in the measurements does not have to be estimated. In addition, an accurate value of the dielectric permittivity of the fabric can be derived without any simulations. The procedure is as follows:

1. Find the fabric of interest, such as denim, felt, or any other fabric type.

2. Measure the sample thickness $(t)$ using an accurate Vernier caliper.

3. A copper tape conductor $\cong 0.035 \mathrm{~mm}$ thick can be used as the ground plane and radiating patch with $(W \times W)$ and $(w \times w)$, respectively, with respect to the assumptions in note 3 from the derived formulas.

4. After fabrication, through a $1 \mathrm{~mm}$ cylinder hole at the center of the unit, connect the inner conductor of the mini-coaxial connector (Hirose H. FL mini-coaxial connector with $50 \Omega$ impedance range) to the center of the square patch and the outer conductor of the mini cable to the ground plane, as it is seen in Fig. 3. ${ }^{(27)}$

5. After calibrating a vector network analyzer $(100 \mathrm{kHz}-12 \mathrm{GHz})$, connect the mini-coaxial cable connector to the calibrated port and sweep to find the resonance frequency by reading $\mathrm{S}_{11}$.

6. Take the lowest resonant frequency and calculate $\varepsilon_{\text {reff }}$ from Eq. (3), then follow the rest of the procedure to derive $\varepsilon_{r}$ from Eq. (5) for the given structure.

7. The method is accurate for a single resonance frequency. The procedure can be repeated with different dimensions of ground plane and patch. Finally, from the extracted values of $\varepsilon_{r}$ at discrete resonance frequencies, the mean value $\varepsilon_{r}$ can be calculated from Eq. (6). 
8. For the purpose of comparison, introduce a new lossless material with a derived $\varepsilon_{r}$ to one of the software for simulating a high-frequency electromagnetic field, such as ANSYS HFSS software or CST Microwave Studio and observe the $\mathrm{S}_{11}$ curve.

\subsection{Advantages}

The proposed method provides a plain and straightforward way to derive the dielectric permittivity of a fabric sample. The derived values of $\varepsilon_{r}$ are accurate to a great extent. In contrast to the approach by Sankaralingam and Gupta, the method in this paper eliminates initial guesses for the dielectric constant of the material being tested. ${ }^{(5)}$ The proposed structure is simple to fabricate and does not require tuning for the feeding point as it is straightforward and directly fed at the center.

\section{Procedure Illustrated Using Examples}

To clarify the proposed method, the rest of this paper covers fabrication and measurements using examples of fabrics, such as felt and nonelastic denim. Measurements for the radiating square patch are carried out for random $(w \times w) \leq(W \times W)$, while the thickness and the area $(W \times W)$ of the fabric and the ground plane are fixed and unchanged. In addition, for a fixed area of $(W \times W)$ and $(w \times w)$, measurements are carried out to observe the effect of the thickness of the fabric on the accuracy of the method. The smaller the size of the square patch, the higher the first resonance frequency. In this case, the operating frequency range of the vector network analyzer and its calibration for error correction should be taken into consideration. Finally, the method is validated using the FR4 substrate widely used in antenna design whose dielectric permittivity is known $\left(\varepsilon_{r}=4.3\right)$.

\subsection{Measurement examples}

In this section, the same dimensions $\left(W \times W=60 \times 60 \mathrm{~mm}^{2}\right.$ and $\left.w \times w=50 \times 50 \mathrm{~mm}^{2}\right)$ are chosen for the geometry presented in Fig. 3 to extract and calculate the permittivity of the sample being tested. The thickness $(t)$ of the felt and denim layers are 1.5 and $1 \mathrm{~mm}$ respectively. The radiating patches are fed at the center in the way previously explained. The area of the fabrics does not have a noticeable effect on the resonance frequency but filling the area of the conductor sheets is required. Therefore, in taking measurements, the sizes of the fabrics being tested are chosen in a random manner. The fabricated units are shown in Fig. 4, and the derived values of both felt and denim are presented in Table 1.

\subsection{Patch size test}

In this section, tests for accuracy are described. The radiating patch area is increased gradually from $\left(w \times w=50 \times 50 \mathrm{~mm}^{2}\right)$ to $\left(w \times w=60 \times 60 \mathrm{~mm}^{2}\right)$, which is the same size as the ground plane shown in Fig. 5. The fabric used in this test is felt with $(1.5 \mathrm{~mm})$ thickness. 


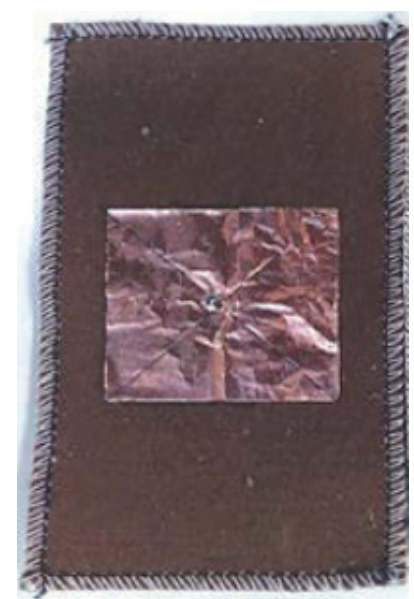

(a)

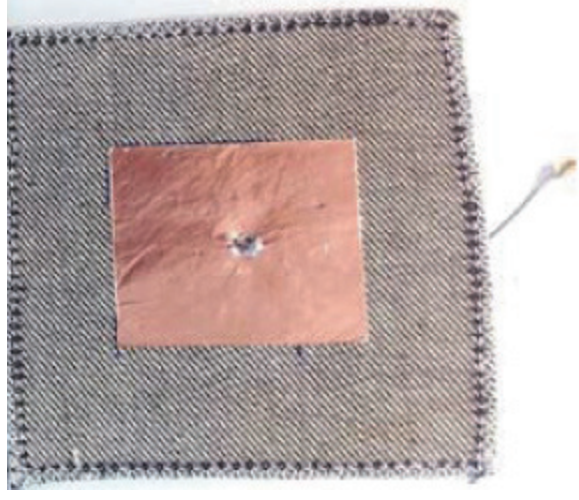

(b)

Fig. 4. (Color online) Fabricated units: (a) felt fabric and (b) denim fabric.

Table 1

Derived values of felt and denim fabrics for the same radiating patch.

\begin{tabular}{lccc}
\hline Fabric type & $\begin{array}{c}\text { Measured first resonance } \\
\text { frequency }\left(f_{r 1}\right)(\mathrm{GHz})\end{array}$ & $\begin{array}{c}\text { Effective dielectric } \\
\text { permittivity }\left(\varepsilon_{r e f f}\right)\end{array}$ & $\begin{array}{c}\text { Extracted dielectric } \\
\text { permittivity }\left(\varepsilon_{r}\right)\end{array}$ \\
\hline Felt & 5.28 & 1.312 & 1.335 \\
Denim & 4.542 & 1.746 & 1.785 \\
\hline
\end{tabular}

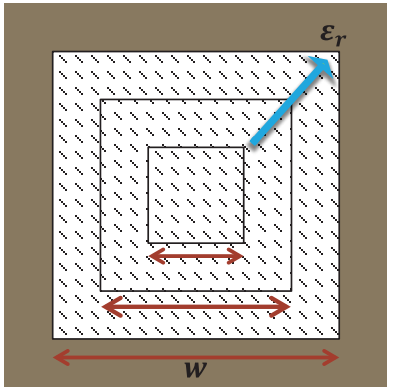

Fig. 5. (Color online) Front view of the unit to test the patch size in the proposed method.

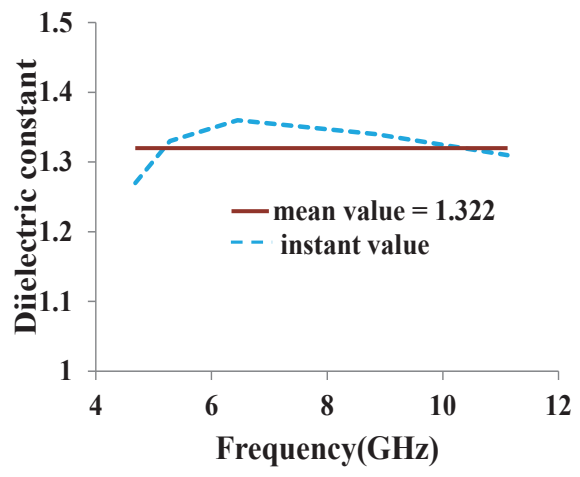

Fig. 6. (Color online) Dispersion characteristics of felt fabric.

As expected, Fig. 6 shows that there are only slight fluctuations in the value of the dielectric constant derived from the measurements. The dielectric constant, which is a function of two interdependent variables, radiating patch area $(w \times w)$ and the corresponding resonance frequency $f_{r 1}$, has a mean value of 1.322 .

\subsection{Fabric thickness test}

In this section, the effect of the sample thickness on the value of the dielectric constant is discussed. As an example of a fabric being tested, denim is shown in Fig. 4(b). Denim fabrics 
with thicknesses of more than $1 \mathrm{~mm}$ are not available commercially. Therefore, double-sided fabric tape is used to attach two or three layers of available non-stretchable denim fabric so that the effect of material thickness may be detected. Table 2 shows that the characteristics of denim are influenced by the tape and its dielectric constant is more dispersed. To confirm that the small deviation in the values of the dielectric constant denim was the result of the tape, more tests were carried out for a single layer of the same denim fabric, $1 \mathrm{~mm}$ thick but having different and random radiating patch sizes. According to the measurements, the value of the dielectric constant of denim remained unchanged around 1.8. Ultimately, new lossless materials with derived $\varepsilon_{r}$ values of the fabrics, denim, and felt were introduced into the software for simulating high-frequency electromagnetic fields, such as ANSYS HFSS software or CST Microwave Studio, and $\mathrm{S}_{11}$ curves of the square microstrip patch units were observed. Good matching in the return loss of the simulated resulted, and the measured results have been observed for the denim and felt fabrics as shown in Figs. 7 and 8 respectively.

\subsection{Validation of the method}

The approval of the proposed method for evaluating the accuracy of the procedure is discussed in this section. In the measurements, an FR4 substrate with a permittivity of 4.3, which is very widely used in electronics, is employed.

The mean value of the derived dielectric constant for the measurements is calculated to show the dispersive characteristics of the substrate in cases of different thicknesses of FR4 as shown in Table 3; the measurement is 4.25. Comparable to the method presented by Paleček et

Table 2

Dispersion characteristics of denim fabric for $\left(w \times w=50 \times 50 \mathrm{~mm}^{2}\right)$ patch size.

\begin{tabular}{lccc}
\hline $\begin{array}{l}\text { Denim thickness }(t) \\
(\mathrm{mm})\end{array}$ & $\begin{array}{c}\text { Measured resonance } \\
\text { frequency in }(\mathrm{GHz})\end{array}$ & $\begin{array}{c}\text { Calculated effective } \\
\text { dielectric constant }\left(\varepsilon_{r e f f}\right)\end{array}$ & $\begin{array}{c}\text { Extracted dielectric } \\
\text { constant }\left(\varepsilon_{r}\right)\end{array}$ \\
\hline 1 & 4.542 & 1.746 & 1.785 \\
2.16 & 4.51 & 1.766 & 1.846 \\
3.34 & 4.405 & 1.834 & 1.95 \\
\hline
\end{tabular}

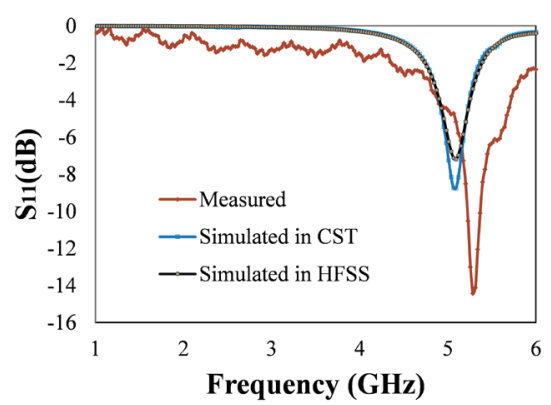

Fig. 7. (Color online) Measured $S_{11}$ vs simulations for denim fabric.

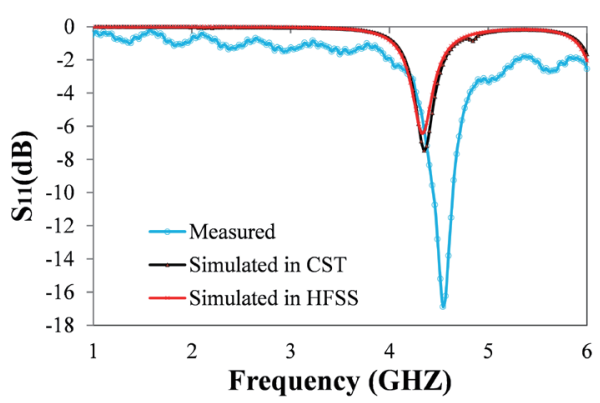

Fig.8. (Color online) Measured $S_{11}$ vs simulations for felt fabric. 
Table 3

Dispersion characteristics of FR4 for $\left(W \times W=60 \times 60 \mathrm{~mm}^{2}\right)$ ground plane size.

\begin{tabular}{lcccc}
\hline $\begin{array}{l}\text { FR4 thickness }(t) \\
(\mathrm{mm})\end{array}$ & $\begin{array}{c}\text { Patch size }(w \times w) \\
\left(\mathrm{mm}^{2}\right)\end{array}$ & $\begin{array}{c}\text { Resonance frequency } \\
(\mathrm{GHz})\end{array}$ & $\begin{array}{c}\text { Effective dielectric constant Dielectric constant } \\
\left(\varepsilon_{\text {reff }}\right)\end{array}$ & $\begin{array}{c}\left(\varepsilon_{r}\right) \\
0.4\end{array}$ \\
$44 \times 44$ & 3.417 & 3.982 & 4.06 \\
0.8 & $44 \times 44$ & 3.364 & 4.107 & 4.26 \\
& $44 \times 44$ & 3.3795 & 4.07 & 4.34 \\
& $52 \times 52$ & 2.8525 & 4.09 & 4.332 \\
\hline
\end{tabular}

al., ${ }^{(28)}$ which was carried out to observe the frequency dependence of the FR4 substrate used in high frequency electronics, the proposed method is quite accurate and requires less effort with minimum knowledge of the material characteristics to calculate the substrate's dielectric constant. In the design of antennas, a tradeoff, which involves both impedance bandwidth and the efficiency of the antenna, is subject to the thickness of the material and should be taken into critical consideration. In this context, one way to enhance the antenna bandwidth is to increase the thickness of the chosen material and change the overall dimensions of the unit slightly.

\section{Applications of Low Loss Fabrics in Smart Clothing}

A newly-introduced concept that involves connecting everything in one network is the Internet of Things (IoT). This trend aims at developing and expanding the capacity of identifying, communicating with and integrating different devices and objects. In this regard, the concept of wearable technologies is rapidly growing and improving. Through these applications and with the aid of wireless, wearable systems, a person in his smart clothes will be able to be part of and stay connected to a core network. ${ }^{(13,29)}$ Therefore, a wearable antenna is an essential component of any wearable wireless system. In this section, the applications of low loss fabrics used in developing flexible and durable antennas are presented.

\subsection{Wearable antenna design process}

Figure 9 presents the process of planar textile antenna design for any wearable applications using fabrics as the substrate.

\subsection{Wearable antenna model}

The structure of the antenna is shown in Fig. 10. The unit is composed of an F-shaped radiating patch antenna backed by a rectangular ground plane with an F-shaped slot in it. The 1-mm-thick low-loss denim fabric characterized in the previous section (see Table 2) was employed as the wearable antenna substrate. The dielectric constant of the denim fabric according to the proposed method is approximately 1.8; it has been added as a new lossless material to the library of CST Microwave Studio for the purposes of modelling and simulation. The detailed dimensions of the textile antenna are presented in Table 4. The F-shaped patch antenna in its upper range of frequencies supports both wireless local area networks (WLAN) 


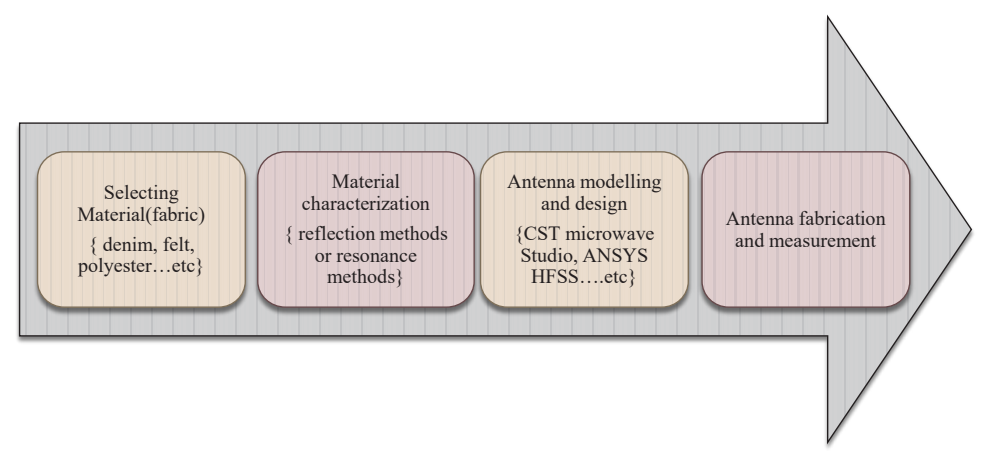

Fig. 9. (Color online) Design process of textile wearable antennas.

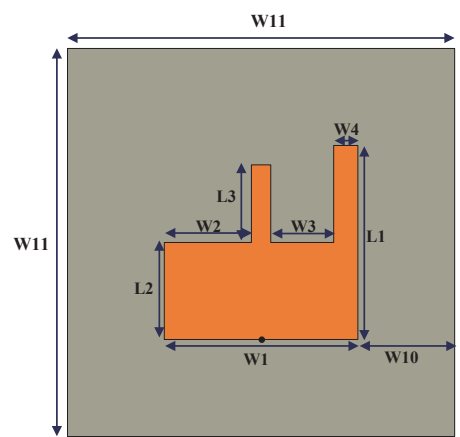

(a)

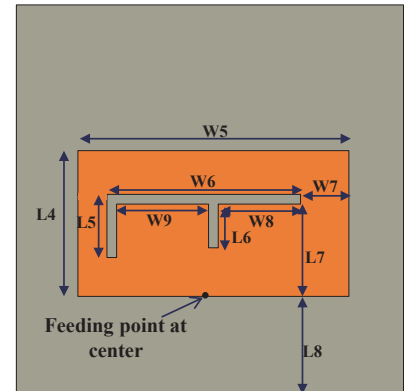

(b)

Fig. 10. (Color online) The structure of the wearable antenna: (a) front view and (b) back view.

Table 4

Details of the antenna dimensions.

\begin{tabular}{lcccccc}
\hline Parameter & W1 & W2 & W3 & W4 & W5 & W6 \\
Physical length (mm) & 40 & 18 & 13 & 5 & 56 & 40 \\
\hline Parameter & W7 & W8 & W9 & W10 & W11 & \\
Physical length (mm) & 10 & 17 & 19 & 20 & 80 & \\
\hline Parameter & L1 & L2 & L3 & L4 & L5 & L6 \\
Physical length (mm) & 40 & 20 & 16 & 30 & 13 & 9 \\
\hline Parameter & L7 & L8 & & & & \\
Physical length (mm) & 19 & 20 & & & & \\
\hline
\end{tabular}

and industrial, scientific, and medical (ISM) radio bands. Figure 11 shows simulated and measured return loss and explain how well the $50 \Omega$ mini-coaxial cable line impedance is matched to the antenna unit. Excellent matching between the simulated and measured $S_{11}$ validates the accuracy of the proposed method to derive the dielectric constant of fabrics for smart clothing and wearable applications. The voltage standing wave ratio (VSWR) of the antenna in the frequency range from 5.6 to $6 \mathrm{GHz}$ is less than 2.0, which implies that less than $12 \%$ of the excited power is reflected back to the feeding line. The polar form radiation pattern of the antenna shows that the antenna has more directivity in both the $X-Z$ and $Y-Z$ planes than in the $X-Y$ plane as shown in Fig. 12. The simulated realized gain of the antenna in direction of the main lobe is $1.98 \mathrm{~dB}$. 


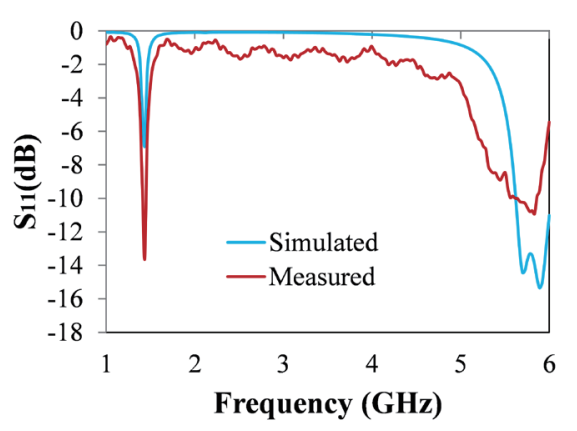

Fig. 11. (Color online) Simulated and measured $\mathrm{S}_{11}$ of the wearable antenna in free space.

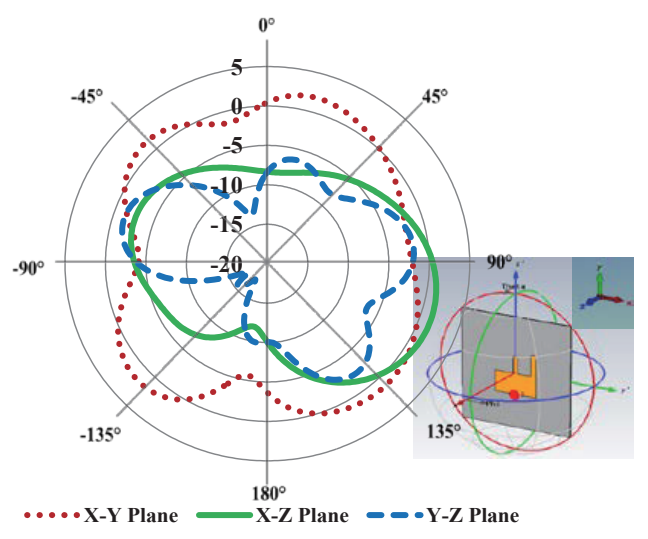

Fig. 12. (Color online) Simulated radiation pattern at $5.75 \mathrm{GHz}$.

\section{Conclusions}

A simple and accurate technique to determine the dielectric constant (real part) of low loss fabrics is proposed in this paper. The structure of the method is a combined model of both a coaxial cable reflection method and a monopole antenna resonance method. Rectangular patch antenna base equations were assets in developing the core equations for this novel technique. The method is an example of applications of antennas to the characterization of materials. The effective relative permittivity of the sample being tested is extracted from the first resonance mode of the patch antenna. Subsequently, dielectric permittivity is calculated by taking the ratio of substrate thickness to patch width into consideration. The technique is unique for its simplicity in calculation, and fabrication as well as in measurements. Previous knowledge of the fabric is not required when applying the method.

The method presented in this paper has been validated in four different forms. First, the size of the radiation square patch was tested within the $w \leq W$ bound. Second, the thickness of the substrate was examined to determine its essential influence on method accuracy. Third, the method was applied to an FR4 substrate with a known relative permittivity of 4.3, in both simulations and actual measurements. Finally, a simple wearable antenna was modeled using CST Microwave Studio based on new denim fabric with a measured dielectric permittivity of 1.8 to show the accuracy of the method for smart clothing applications. It was determined that the method is accurate, stable, and representative. Owing to the presence of air gaps between the fibers of fabric, heterogeneity was introduced; therefore, the value of the dielectric permittivity of the fabric was bounded between 1 and 2. The method is only designed to derive the real part of dielectric constant of sample fabrics, assuming that the fabric is low loss or loss free.

\section{References}

1 C. Loss, R. Gonalves, C. Lopes, P. Pinho, and R. Salvado: Sensors 16 (2016) 938.

2 I. Locher, M. Klemm, T. Kirstein, and G. Tröster: IEEE Trans. Adv. Packag. 29 (2006) 777. 
3 R. Salvado, C. Loss, Gon, and P. Pinho: Sensors 12 (2012) 15841.

4 L. F. Chen, C. K. Ong, C. P. Neo, V. V. Varadan, and V. K. Varadan: Microwave Electronics (John Wiley \& Sons, Chichester, 2004) p. 37

5 S. Sankaralingam and B. Gupta: IEEE Trans. Instrum. Meas. 59 (2010) 3122.

6 H. Henson and J. L. Sexton: Microwave Electronics (John Wiley \& Sons, Chichester, 2004) p. 142

7 J. Heinola, K. Lätti, P. Silventoinen, J. Ström, and M. Kettunen: Proc. 2004 IEEE 9th Int. Symp. Advanced Packaging Materials (IEEE, 2004) 235.

8 U. C. Hasar: IEEE Microw. Wirel. Components Lett. 19 (2009) 801.

9 U. C. Hasar and C. R. Westgate: IEEE Trans. Microw. Theory Tech. 57 (2009) 471.

10 H. Zhou, G. Lu, Y. Li, S. Wang, and Y. Wang: Proc. 2009 PIERS (PIERS 2009) 768.

11 L. F. Chen, C. K. Ong, C. P. Neo, V. V. Varadan, and V. K. Varadan: Microwave Electronics (John Wiley \& Sons, Chichester, 2004) p. 175.

12 J. S. Bobowski and T. Johnson: Prog. Electromagn. Res. 40 (2012) 159.

13 S. Wang, M. Niu, and D. Xu: IEEE Trans. Microw. Theory Tech. 46 (1998) 2145.

14 S. Seewattanapon and P. Akkaraekthalin: J. Electromagn. Anal. Appl. 3 (2011) 312.

15 A. Ćenanović, M. Schramm, and L. P. Schmidt: 2011 IEEE MTT-S Int. Microwave Symp. Digest (IEEE, 2011$) 1$.

16 G. S. Smith and J. D. Nordgard: IEEE Trans. Antennas Propag. 33 (1985) 783.

17 E. Odelstad, S. Raman, A. Rydberg, and R. Augustine: IEEE J. Transl. Eng. in Health and Medicine 2 (2014) 1.

18 L. F. Chen, C. K. Ong, C. P. Neo, V. V. Varadan, and V. K. Varadan: Microwave Electronics (John Wiley \& Sons, Chichester, 2004) p. 208.

19 S. Zhu and R. Langley: IEEE Trans. Antennas Propag. 57 (2009) 926.

20 S. Yan, P. J. Soh, and G. A. E. Vandenbosch: IEEE Antennas Wirel. Propag. Lett. 14 (2015) 1486.

21 V. Karthik and T. R. Rao: Proc. 2015 Int. Conf. Comput. Commun. Technol. (ICCCT, 2012) 221.

22 J. Trajkovikj and A. K. Skrivervik: IEEE Antennas Wirel. Propag. Lett. 14 (2015) 1530.

23 S. Yan, P. J. Soh, and G. A. E. Vandenbosch: IEEE Trans. Antennas Propag. 63 (2015) 4165.

24 H. C. Yang, H. I. Azeez, C. K. Wu, and W. S. Chen: 2017 IEEE Int. Conf. Computational Electromagnetics (ICCEM, 2017) 185.

25 H. R. Raad, A. I. Abbosh, H. M. Al-Rizzo, and D. G. Rucker: IEEE Trans. Antennas Propag. 61 (2013) 524.

26 C. A. Balanis: Microstrip Antennas (John Wiley \& Sons, New Jersey, 2005) p. 811.

27 Global sources: Homepage of global sources, http://www.globalsources.com/gsol/I/Coaxial-cable/p/ sm/1131705260.htm\#1131705260 (accessed July 2017).

28 J. Paleček, M. Vestenický, P. Vestenický, and J. Spalek: 2013 12th IFAC Conf. Programmable Devices and Embedded Systems (IFAC, 2013) 90.

29 B. Gupta, S. Sankaralingam, and S. Dhar: 2010 10th Mediterranean Microwave Symp. (MMS, 2010) 251. 